\title{
Isolated Angiitis of the Central Nervous System First Presenting as Intracranial Hemorrhage during Cesarean Section
}

\author{
Yuzuru Yasuda, Isao Matsuda, Yu KanG*, Tatsuyoshi SAIGA** and Masakuni KameYama*** \\ A case of isolated angiitis of the central nervous system (CNS) which first presented as \\ intracranial hemorrhage during cesarean section is reported. Only one case of isolated angiitis of \\ the CNS in post partum has been reported to date. Although a headache in labor and puerperium \\ usually suggests subarachnoid hemorrhage, isolated angiitis of the CNS should be considered in \\ the differential diagnosis.
} (Internal Medicine 32: 745-748, 1993)

Key words: Pregnancy, corticosteroid, cyclophosphamide

\section{Introduction}

Isolated angiitis of the central nervous system (CNS) is an uncommon entity with a lesion which is restricted to small and medium size arteries and venules of the brain and spinal cord (1, 2). The prognosis was previously considered poor, but there are several reports suggesting that treatment with corticosteroids plus cyclophosphamide is effective $(1,3,4)$.

This case first presented as intracranial hemorrhage during cesarean section. To date, only one case of isolated angiitis of the CNS in postpartum has been reported (5). When persistent headache occurs in labor and puerperium, isolated angiitis of the CNS should be considered in the differential diagnosis.

\section{Case Report}

A 27 -year-old female, para 0 , gravida 1 , was admitted at 40 week's gestation for cesarean section, because of cephalopelvic disproportion. She had no symptoms suggestive of preeclampsia. During the operation she developed severe hypertension (220/ $110 \mathrm{mmHg}$ ), but her blood pressure returned to normal after the operation. After she awoke from the operation, she complained of a severe headache. No muscle weakness or sensory disturbance was seen. The headache persisted for 10 days after the operation. She was then referred to our hospital because of blurred vision and persistent slight fever following the operation.

The patient was alert, but calculation was slightly disturbed. Eye movement was normal with no anisocoria. Light reaction was prompt and no hemianopsia was detected. Other cranial nerves were also normal. She had no hemiparesis. Deep tendon reflex was normal and no pathologic reflex was seen. There was no superficial or deep sensory disturbance. Stereoagnosia was not seen. Signs of meningeal irritation or extrapyramidal dysfunction were absent. There was no dressing apraxia, constructional apraxia, ideational apraxia or unilateral spatial neglect. but slight topographical amnesia was present.

On admission, a complete blood count, routine blood tests, coagulation studies, luetic serology, blood culture and urinalysis were unremarkable. C-reactive protein and rheumatoid factor were negative. Antinuclear antibodies, anti-DNA antibodies, serum immunoglobulin levels, complement levels and angiotensin-converting enzyme were normal. However, circulating immune complex and ESR were elevated to $4.1 \mu \mathrm{g} / \mathrm{dl}$ (normal: below $3.0 \mu \mathrm{g} / \mathrm{dl}$ ) and $35 \mathrm{~mm} / \mathrm{hr}$, respectively. Herpes simplex, herpes zoster, cytomegalovirus, mycoplasma, and toxoplasma titer were all normal in serum and cerebrospinal fluid (CSF). CSF analysis revealed an opening pressure of $120 \mathrm{~mm}$ water, 1 WBC (lymphocyte)/cu mm, a protein concentration of $26 \mathrm{mg} /$ $\mathrm{dl}$ and a glucose level of $54 \mathrm{mg} / \mathrm{dl}$. Cultures of CSF were negative. There were no abnormal findings in electromyogram, peripheral nerve conduction velocity, or EEG. CT scan disclosed a hematoma in the right parieto-occipital lobe (Fig. 1). Carotid and vertebral angiogram demonstrated areas of stenosis and irregularity of intracranial arteries (Fig. $2 \mathrm{ABC}$ ). There was no abnormality in the branches of external carotid arteries. Systemic angiogram showed no evidence of systemic vasculitis. Biopsy of the temporal artery was normal.

Treatment with prednisolone was started immediately after admission; $60 \mathrm{mg} /$ day for 2 weeks and $50 \mathrm{mg} /$ day for 1 week. ESR decreased to $10 \mathrm{~mm} / \mathrm{hr}$, but slight fever continued and the second carotid and vertebral angiogram showed no improve-

From the Department of Neurology, *Neurosurgery and **Pathology, Otsu Red Cross Hospital, Otsu and ***Department of Neurology, Sumitomo Hospital, Received for publication April 9, 1993; Accepted for publication September 29, 1993

Reprint requests should be addressed to Dr. Yuzuru Yasuda, the Department of Neurology, Otsu Red Cross Hospital, 1-1-35, Nagara, Otsu 520 


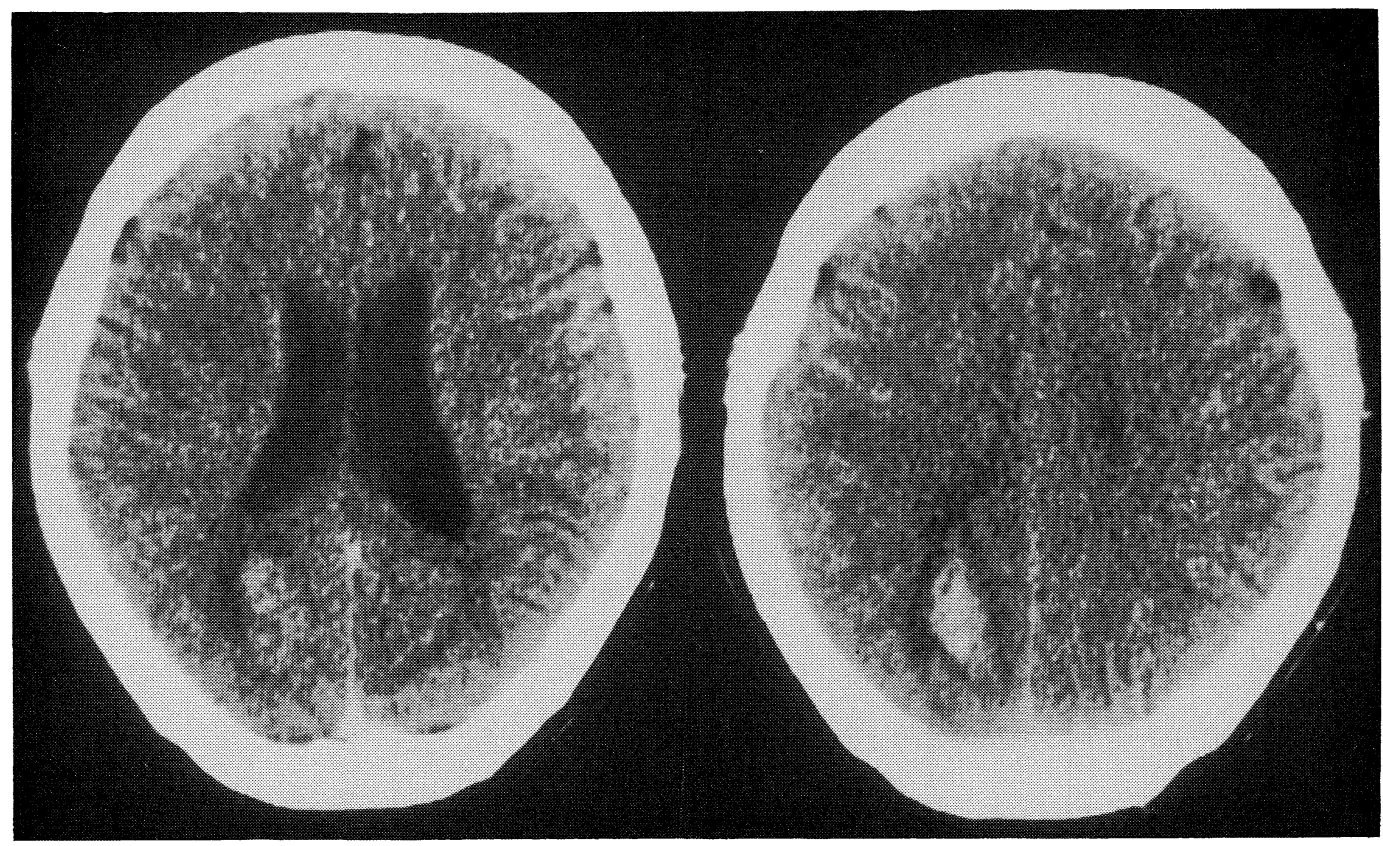

Fig. 1. CT scan disclosed a hematoma in the right parieto-occipital lobe.

ment. Therefore treatment with prednisolone (40 mg every other day) plus cyclophosphamide ( $100 \mathrm{mg} /$ day) was carried out. Circulating immune complex on the 30th and 58th day after treatment with prednisolone and cyclophosphamide became 3.5 and $1.9 \mu \mathrm{g} / \mathrm{ml}$, respectively. Angiogram on the 56th and 330th day after treatment with prednisolone and cyclophosphamide showed a marked improvement of angiitis (Fig. 2 DEF). She continued to be healthy without any neurological defects for 15 months. Prednisolone was tapered and cyclophosphamide was stopped. Thereafter she complained of headache and showed slight fever again. ESR and circulating immune complex were elevated $(25 \mathrm{~mm} / \mathrm{hr}, 3.7 \mu \mathrm{g} / \mathrm{ml}$, respectively). Prednisolone was increased and cyclophosphamide was administered again. She became afebrile, and ESR and circulating immune complex was normalized $(9 \mathrm{~mm} / \mathrm{hr}, 2.0 \mu \mathrm{g} /$ $\mathrm{ml}$, respectively) 1 month later.

\section{Discussion}

This patient first presented as intracranial hemorrhage during cesarean section and showed no findings suggestive of autoimmune disease except for high immune complex titer. Neurologically, she showed only headache, blurred vision and slight topographical amnesia which is concerned with the small size of right parieto-occipital lobe hematoma. Carotid and vertebral angiogram demonstrated striking areas of stenosis and irregularity of all intracranial arteries and systemic angiogram showed no evidence of systemic vasculitis. As the lesion was confined to the intracranial arteries and no factor which induces vasculitis, for example, infections, malignancy, autoimmune diseases and chemical factors, was not present, this case can be diagnosed as isolated angiitis of the CNS $(1,2)$.

Isolated angiitis of the CNS was previously considered as a fatal disease $(1,2)$. Several reports showed that the disease progressed despite treatment with corticosteroids $(6,7)$ or corticosteroid and azathioprine (7). But some patients showed a transient improvement with corticosteroids, and required the addition of the cyclophosphamide to maintain the remission (1). A recent study showed that treatment with a combination of cyclophosphamide and corticosteroids is effective; Moore reported 5 cases who showed improvement, and recommended the following treatment regimens for the initial 6 weeks of therapy: 1) prednisone 40 to $60 \mathrm{mg} / \mathrm{day}$, and 2) cyclophosphamide $100 \mathrm{mg} /$ day (3). Moreover, Vanderzant et al reported a case who showed improvement with cyclophosphamide plus corticosteroid (4). The present case did not improve with corticosteroid alone, but showed marked improvement with cyclophosphamide plus corticosteroid. Treatment with a combination of cyclophosphamide plus corticosteroid is rewarding in isolated angiitis of the CNS.

To date, only one case of isolated angiitis of the CNS in the postpartum has been reported. The case in the report of Langlois et al (5) 1 month postpartum, developed a severe headache associated with subarachnoid hemorrhage and was treated with corticosteroid alone. She showed improvement temporarily, but resulted in a flare later because cyclophosphamide was not administered. The present case also showed a severe headache during cesarean section associated with cerebral hemorrhage, and responded to cyclophosphamide plus corticosteroid. But she showed slight fever and elevation of ESR and circulating immune complex again by tapering of corticosteroid and cessation of cyclophosphamide, and showed 


\section{Isolated Angiitis of the CNS}

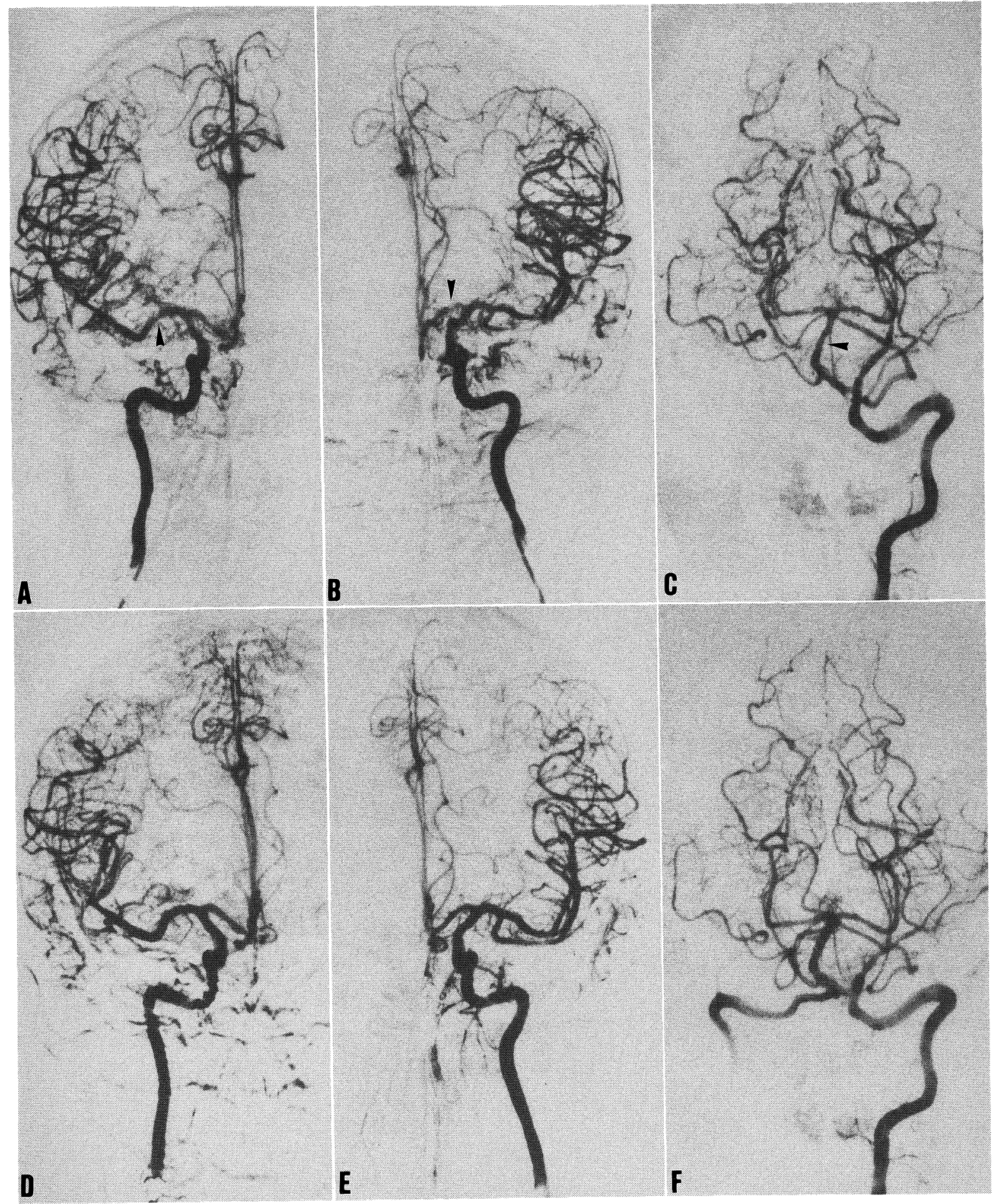

Fig. 2. Carotid (A, D; right, B, E; left) and vertebral (C, F) angiogram showed striking areas of stenosis (arrowhead) and irregularity of many intracranial branches before treatment. After treatment marked improvement of the angiitis was seen (A, B, C; before treatment, D, E, F; after treatment). 


\section{YASUDA et al}

improvement again by readministration of corticosteroid and cyclophosphamide. Therefore our case can be differentiated from peripartum cerebral angiopathy whose etiology is considered as vasoconstriction $(8,9)$. Moreover, isolated angiitis of the CNS in a young female on the contraceptive (10) has been reported. As it is well known that estrogens alter plasma proteins making blood more coagulable, it is possible that a female hormone may induce isolated angiitis of the CNS.

From now on isolated angiitis of the CNS should be considered in the differential diagnosis of intrapartum and postpartum headache.

\section{References}

1) Cupps TR, Moore PM, Fauci AS. Isolated angiitis of the central nervous system. Prospective diagnostic and therapeutic experience. Am J Med 74: 97, 1983.

2) Moore PM, Cupps TR. Neurological complications of vasculitis. Ann
Neurol 14: 155, 1983.

3) Moore PM. Diagnosis and management of isolated angiitis of the central nervous system. Neurology 39: 167, 1989.

4) Vanderzant C, Bromberg M, MacGuire A, McCune WJ. Isolated smallvessel angiitis of the central nervous system. Arch Neurol 45: 683, 1988.

5) Langlois PF, Sharon GE, Gawryl MS. Plasma concentrations of comple ment-activation complexes correlate with disease activity in patients diagnosed with isolated central nervous system vasculitis. J Allergy Clin Immunol 83: 11, 1989.

6) Harrison PE Jr. Granulomatous angiitis of the central nervous system: Case report and review. J Neurol Sci 29: 335, 1976.

7) Griffin J, Price DL, Davis L, McKhann GM. Granulomatous angiitis of the central nervous system with aneurysms of multiple cerebral arteries. Trans Am Neurol Assoc 98: 145, 1973.

8) Brick JF. Vanishing cerebrovascular disease of pregnancy. Neurology 38: 804, 1988.

9) Bogousslavsky J, Despland PA, Regli F, Dubuis PY. Postpartum cerebral angiopathy: Reversible vasoconstriction assessed by transcranial doppler ultrasounds. Eur Neurol 29: 102, 1989.

10) Nagaratnam $N$, James WE. Isolated angiitis of the brain in a young female on the contraceptive pill. Postgrad Med J 63: 1085, 1987. 